\title{
On-orbit Calibration and Performance of Aqua MODIS Reflective Solar Bands
}

\author{
Xiaoxiong Xiong ${ }^{\mathrm{a}}$, Junqiang Sun ${ }^{\mathrm{b}}, \mathrm{Xiaobo}^{\mathrm{X}} \mathrm{e}^{\mathrm{b}}$, William Barnes ${ }^{\mathrm{c}}$, and Vincent \\ Salomonson $^{\mathrm{d}}$ \\ ${ }^{\mathrm{a}}$ Sciences Exploration Directorate, NASA/GSFC, Greenbelt, MD 20771; \\ ${ }^{\mathrm{b}}$ Science Systems and Applications, Inc., 10210 Greenbelt Road, Lanham, MD 20706; \\ ${ }^{c}$ University of Maryland, Baltimore County, 1000 Hilltop Circle, Baltimore, MD 21250. \\ ${ }^{\mathrm{d}}$ University of Utah, Salt Lake City, UT 84112
}

\begin{abstract}
Aqua MODIS has successfully operated on-orbit for more than 6 years since its launch in May 2002, continuously making global observations and improving studies of changes in the Earth's climate and environment. 20 of the 36 MODIS spectral bands, covering wavelengths from 0.41 to $2.2 \mu \mathrm{m}$, are the reflective solar bands (RSB). They are calibrated on-orbit using an on-board solar diffuser (SD) and a solar diffuser stability monitor (SDSM). In addition, regularly scheduled lunar observations are made to track the RSB calibration stability. This paper presents Aqua MODIS RSB on-orbit calibration and characterization activities, methodologies, and performance. Included in this study are characterizations of detector signal-to-noise ratio (SNR), short-term stability, and long-term response change. Spectral wavelength dependent degradation of the SD bidirectional reflectance factor (BRF) and scan mirror reflectance, which also varies with angle of incidence (AOI), are examined. On-orbit results show that Aqua MODIS onboard calibrators have performed well, enabling accurate calibration coefficients to be derived and updated for the Level 1B (L1B) production and assuring high quality science data products to be continuously generated and distributed. Since launch, the short-term response, on a scan-by-scan basis, has remained extremely stable for most RSB detectors. With the exception of band 6, there have been no new RSB noisy or inoperable detectors. Like its predecessor, Terra MODIS, launched in December 1999, the Aqua MODIS visible (VIS) spectral bands have experienced relatively large changes, with an annual response decrease (mirror side 1) of $3.6 \%$ for band 8 at $0.412 \mu \mathrm{m}, 2.3 \%$ for band 9 at $0.443 \mu \mathrm{m}, 1.6 \%$ for band 3 at $0.469 \mu \mathrm{m}$, and $1.2 \%$ for band 10 at $0.488 \mu \mathrm{m}$. For other RSB bands with wavelengths greater than $0.5 \mu \mathrm{m}$, the annual response changes are typically less than 0.5\%. In general, Aqua MODIS optics degradation is smaller than Terra MODIS and the mirror side differences are much smaller. Overall, Aqua MODIS RSB on-orbit performance is better than Terra MODIS.
\end{abstract}

Keywords: Aqua, MODIS, reflective solar bands, calibration, solar diffuser, solar diffuser stability monitor, SD degradation, SNR 


\section{INTRODUCTION}

The Aqua mission is a major component of NASA's Earth Observing System (EOS), carrying a total of six Earth-observing instruments [1]. It was launched on May 4, 2002 in a near sun-synchronous polar orbit with a nominal altitude of $705 \mathrm{~km}$ and an equatorial crossing time of 1:30PM (ascending northwards). MODIS is the keystone instrument onboard the Aqua spacecraft, and is also one of the two instruments currently operated on both the Terra and Aqua spacecrafts, with Clouds and the Earth's Radiant Energy System (CERES) being the other. Developed with improvements over its heritage sensors, MODIS observations are made in a broad spectral range: from visible (VIS) to long-wave infrared (LWIR), a wide field of view (FOV): $\pm 55^{\circ}$ from instrument nadir, and three spatial resolutions: $0.25 \mathrm{~km}$ ( 2 bands), $0.5 \mathrm{~km}$ ( 5 bands), and $1 \mathrm{~km}$ (29 bands) [2-3]. Because of this, MODIS data products have provided many science applications for monitoring changes in the Earth's land, oceans, and atmosphere, and associated impact on the Earth's climate and life. With improved spatial and spectral resolutions and frequent global coverage, MODIS observations have significantly extended and enhanced existing data records from heritage sensors [4-7]. MODIS was built by Raytheon Santa Barbara Remote Sensing (SBRS) located at Goleta, California (recently moved to Raytheon Space and Airborne Systems at El Segundo, California). Its on-orbit operation is managed by the Flight Operation Team (FOT) of EOS Science Mission Operations (ESMO) and calibration is performed by the MODIS Characterization Support Team (MCST) at NASA/GSFC.

MODIS has 20 reflective solar bands (RSB) with wavelengths from 0.41 to $2.2 \mu \mathrm{m}$ and 16 thermal emissive bands (TEB) from 3.7 to $14.4 \mu \mathrm{m}$. It is a cross-track scanning radiometer, which collects data using a two-sided scan mirror and produces a swath $10 \mathrm{~km}$ along-track (at nadir) by $2330 \mathrm{~km}$ cross-track each $1.478 \mathrm{sec}$. scan. This enables complete global coverage in less than 2 days. In order to maintain its on-orbit calibration accuracy and, therefore, the science product quality, MODIS was built with a number of onboard calibrators (OBC). Figure 1 is a schematic of the MODIS scan cavity and its onboard calibrators, which include a solar diffuser (SD), a solar diffuser stability monitor (SDSM), a v-grooved blackbody (BB), and a spectro-radiometric calibration assembly (SRCA). Normally, the SD and SDSM are used together for RSB calibration and the BB for TEB calibration. The SRCA is designed primarily for sensor spectral (RSB only) and spatial (RSB and TEB) characterization with limited capability of monitoring RSB radiometric calibration stability [8-13].

This paper presents Aqua MODIS RSB on-orbit calibration and characterization over its mission from launch to present. It focuses on applications and results from sensor OBC data sets and illustrates RSB radiometric performance. The sensor spatial and spectral characterization issues are not covered in this paper. A complementary summary of Aqua MODIS TEB on-orbit calibration and performance has been reported in a separate paper [14]. Section 2 of this paper provides a brief description of sensor pre-launch calibration background for Aqua MODIS RSB and its on-board calibration system. It is followed by an overview of RSB on-orbit calibration algorithms which utilize SD/SDSM and lunar observations, and associated operational activities in section 3. Section 4 presents Aqua 
MODIS RSB performance with results derived from instrument on-orbit measurements. Issues that could impact RSB calibration accuracy and data product quality, such as SD $\mathrm{BRF}$ and scan mirror reflectance on-orbit degradation, are discussed. Provided in section 5 are comparisons of Terra and Aqua MODIS RSB performance and a list of lessons learned from their on-orbit operation and calibration. Section 6 is a summary of this paper. On-orbit results show that Aqua MODIS RSB on-board calibrators have continued to effectively monitor SD degradation and track RSB responses, thus providing useful data sets to produce and update calibration coefficients. The annual SD (BRF) degradation rate of Aqua MODIS is approximately $2.6 \%$ at $0.412 \mu \mathrm{m}, 1.6 \%$ at $0.466 \mu \mathrm{m}$, and $1.0 \%$ at $0.530 \mu \mathrm{m}$. This is similar to Terra MODIS when operated under similar conditions. The largest RSB response decrease (mirror side 1) is $3.6 \%$ per year for band 8 at $0.412 \mu \mathrm{m}$, followed by $2.3 \%$ for band 9 at $0.443 \mu \mathrm{m}$ and $1.6 \%$ for band 3 at $0.469 \mu \mathrm{m}$. In general, changes in Aqua MODIS RSB responses and, particularly, their mirror side differences are smaller than Terra MODIS. With the exception of band 6, a known problem since pre-launch, there have been no new noisy or inoperable RSB detectors after more than 6 years of on-orbit operations.

\section{PRE-LAUNCH CALIBRATION AND ON-BOARD CALIBRATORS}

\subsection{MODIS RSB}

For reference purposes, Table 1 provides a summary of reflective solar bands (RSB) key design specifications, including center wavelengths $(\mathrm{CW})$, bandwidths (BW), spatial resolutions in terms of instantaneous field of view (IFOV) at nadir, spectral band locations on the focal plane assemblies (FPA), typical and maximum scene radiances (Ltyp amd Lmax), and detector signal-to-noise ratios (SNR) at Ltyp. Data products derived from observations made by MODIS RSB can be applied to studies of the land/cloud/aerosols boundaries and properties, ocean color and biogeochemistry, atmospheric water vapor, and cirrus clouds. MODIS spectral bands are located on the FPA according to their spectral wavelengths. Bands $8,9,3,10-12$, and 4, with center wavelengths from 0.41 to $0.56 \mu \mathrm{m}$, are located on the visible (VIS) FPA; bands 1, 13-15, 2 , and 16 , from 0.65 to $0.87 \mu \mathrm{m}$, are on the near-infrared (NIR) FPA; and the short-wave infrared (SWIR) bands 5-7 and 26, with wavelengths above $1.2 \mu \mathrm{m}$, are co-located with the mid-wave infrared (MWIR) bands on the short- and mid-wave infrared (SMIR) FPA. There are 40, 20, and 10 detectors for each $0.25 \mathrm{~km}$ (bands $1-2$ ), $0.5 \mathrm{~km}$ (bands $3-7$ ), and $1 \mathrm{~km}$ (bands 8-19 and 26) spatial resolution band, respectively. Bands 13 and 14 are the time-delay and integration (TDI) bands, with each using a pair of 10-detector arrays. Together, there are a total of 330 individual RSB detectors.

In addition to SNR requirements shown in Table 1, the RSB radiometric calibration requirements are $\pm 2 \%$ for the reflectance factors and $\pm 5 \%$ for the radiances. These requirements, also specified at typical scene radiances, apply only to observations made at scan angles within $\pm 45^{\circ}$. For non-typical scene radiances from 0.3Ltyp to $0.9 \mathrm{Lmax}$, the calibration requirements are relaxed by an additional $1 \%$. Due to specifically defined and stringent calibration requirements, each MODIS (Terra/Aqua) went through extensive pre-launch calibration and characterization activities. Once operated on-orbit, the sensor 
performance is continuously monitored using its on-board calibrators. MODIS on-orbit calibration capability is one of the most improved features over its heritage sensors.

\subsection{Pre-launch Calibration}

Aqua MODIS RSB pre-launch calibration measurements, made in the thermal vacuum (TV) environment, were specifically designed to characterize each detector's gain, dynamic range, response nonlinearity, and signal-to-noise ratio (SNR). The measurements were made at different instrument or TV environment temperatures in order to compute detector temperature correction coefficients needed for on-orbit applications. From pre-launch calibration and characterization, Aqua MODIS RSB demonstrated better overall performance than its predecessor, Terra MODIS, with an exception of band 6, which had 11 inoperable detectors and a few noisy detectors. Other key calibration parameters, such as sensor response versus scan-angle (RVS), relative spectral responses (RSR), and the polarization sensitivity, were derived from systemlevel measurements at instrument ambient in a clean-room environment.

Like Terra MODIS, the SWIR bands (5-7 and 26) of Aqua MODIS also experienced outof-band (OOB) thermal leaks and electronic crosstalk. Extensive pre-launch efforts were made by the instrument vendor to characterize and mitigate these effects. Consequently, the magnitude of the thermal and electronic crosstalk was much smaller in Aqua MODIS SWIR bands, which led to better data product quality [15].

One of the critically important pre-launch calibration tasks for the RSB was to fully characterize the bi-directional reflectance factor (BRF) of its on-board SD panel. This characterization was designed to establish a link between on-orbit calibration and groundbased reference. It was performed by the instrument vendor at the SD component level, with measurements made in a comparative mode using reference samples traceable to NIST (National Institute of Standards and Technology) reflectance standards. The measurements were made at seven wavelengths $(0.4 \mu \mathrm{m}, 0.5 \mu \mathrm{m}, 0.6 \mu \mathrm{m}, 0.7 \mu \mathrm{m}, 0.9 \mu \mathrm{m}$, $1.1 \mu \mathrm{m}$, and $1.7 \mu \mathrm{m}$ ) and at nine illuminating directions (a combination of 3 elevation angles and 3 azimuth angles). From these measurements, a 2-dimensioanl quadratic fitting was made over illuminating/viewing geometries at each wavelength and a linear interpolation of the SD BRF profiles was made with wavelengths to match RSB spectral bands $[10,16]$.

\subsection{On-board SD and SDSM}

The MODIS RSB on-board radiometric calibration system consists of a solar diffuser (SD) and a solar diffuser stability monitor (SDSM). The SD panel, made of space-grade Spectralon, provides a full aperture calibration target with the same optical path as the Earth view (EV) for each detector. The SD BRF, as described in 2.2, was characterized pre-launch, and its on-orbit degradation is tracked by the SDSM, which is operated during each SD calibration event. The SDSM is effectively, by itself, a ratioing radiometer. It consists of a solar integration sphere (SIS) with 9 detectors covering wavelengths from 0.41 to $0.94 \mu \mathrm{m}$, as shown in Table 2. Also listed in Table 2 are the closely matched MODIS bands. A rotating optical system in the SDSM provides alternate measurements of direct sunlight and sunlight diffusely reflected from the SD panel, and a 
dark view for measuring SDSM detector offsets. The time series of the ratios of each SDSM detector's SD view response to the Sun view response, corrected for the viewing geometry and background, provides a trend of SD on-orbit degradation. The SDSM assembly also includes a fixed screen in its Sun view path. It was designed to match the SDSM responses between its Sun view and SD view [17].

MODIS spectral bands were designed with different gains to optimize their specified applications. The ocean color bands 8-16 have higher gains than other RSB bands and their responses saturate when viewing the $\mathrm{SD}$ with direct solar illumination. To overcome this limitation, a retractable solar diffuser screen (SDS) was built as part of the SD door assembly, such that the SD calibration can be made with and without the SDS in place. The SD door covers both the SD and SDSM views to the Sun. It is normally closed when there is no SD/SDSM calibration event. In addition to the on-board SD and SDSM, an instrument space view port provides system level measurements of each detector's offset or background. There are 50 data samples collected for each scan over the SD and SV calibration sectors for $1 \mathrm{~km}$ resolution bands. For $0.25 \mathrm{~km}$ and $0.5 \mathrm{~km}$ bands, there are 2 and 4 sub-samples, accordingly, to match each $1 \mathrm{~km}$ data sample.

\section{CALIBRATION ALGORITHMS AND ACTIVITIES}

\subsection{RSB Calibration Algorithms}

MODIS RSB radiometric calibration is reflectance based using a solar diffuser. The Earth view scene top-of-atmosphere (TOA) reflectance factors are measured with reference to the SD bi-directional reflectance factor (BRF). The calibration coefficients (inverse gains), $m_{l}$, are computed offline from regularly-scheduled SD and SDSM observations and updated as needed in the Level 1B (L1B) algorithm (or code) via time dependent look-up tables (LUTs). For a given band, detector, sub-sample (for sub-kilometer resolution bands 1-7), and mirror side, the $m_{l}$ can be expressed by

$$
m_{1}=\frac{\rho_{S D} \cos \left(\theta_{S D}\right)}{d n_{S D}^{*} \cdot d_{E S(S D)}^{2}} \cdot \Gamma_{S D S} \cdot \Delta_{S D}
$$

where $\rho_{\mathrm{SD}}$ is the $\mathrm{SD}$ initial $\mathrm{BRF}$ derived from pre-launch measurements, $\theta_{S D}$ is the SD solar zenith angle, $\left(\mathrm{dn}_{\mathrm{SD}}\right)^{*}$ is the detector response to the $\mathrm{SD}$ in digital number with corrections applied to remove the effects due to instrument background, temperature, and viewing angles, and $\mathrm{d}_{\mathrm{ES}(\mathrm{SD})}$ is the Earth-Sun distance in $\mathrm{AU}$ at the time of the $\mathrm{SD}$ observation. A vignetting function, $\Gamma_{S D S}$, is included in Eqn. 1 for the bands calibrated with the SD screen (SDS) in place. Otherwise $\Gamma_{S D S}$ is set to 1 . Finally, a SD degradation factor, $\Delta_{\mathrm{SD}}$, needs to be applied to remove the impact due to SD BRF on-orbit changes.

Using the same approach and replacing the SD subscript in Eqn. 1 with EV, the Earth view $(\mathrm{EV})$ reflectance factor, $\rho_{\mathrm{EV}} \cos \left(\theta_{\mathrm{EV}}\right)$, can be calculated by

$$
\rho_{E V} \cos \left(\theta_{E V}\right)=m_{1} \cdot d n_{E V}^{*} \cdot d_{E S(E V)}^{2}
$$


For obvious reasons, the SDS vignetting function and SD degradation factor are not needed in Eqn. 2 for the EV observations. MODIS L1B also provides TOA EV radiances for the RSB. The radiances are converted from the reflectance factors by multiplying a factor of $E_{S U N} / \pi$, with $E_{S U N}$ being the solar irradiance weighted by each detector's relative spectral response (RSR). An additional Earth-Sun distance factor is also applied to the conversion, since the reflectance factor is not Earth-Sun distance dependent. The solar irradiance model used for MODIS (Terra and Aqua) RSB is a combination of (1)

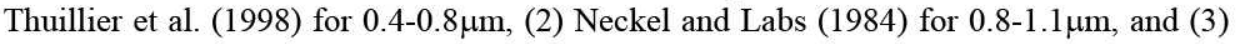
Smith and Gottlieb (1974) for wavelengths above 1.1 $\mu \mathrm{m}$ [10].

For SWIR bands, an additional correction algorithm is applied to both SD and EV detector responses. These correction coefficients are derived and monitored from SWIR bands nighttime observations [15]. More details on MODIS RSB calibration algorithms and reflectance and radiance products can be found on the MODIS calibration web site (http://www.mcst.ssai.biz/mcstweb/index.html).

\subsection{Calibration Activities}

Normally, each complete RSB calibration consists of 2 sets of SD observations, which are implemented in 2 consecutive orbits: one with the SDS in front of the SD panel and one without the SDS. For each SD calibration event, the on-board SDSM is also operated in order to track on-orbit changes in SD BRF. Although SD calibration opportunity exists in every orbit, the SD/SDSM calibration event or activity is scheduled on a less frequent basis. As previously mentioned, the SD aperture door is closed if there is no SD/SDSM calibration event, in order to prevent direct sunlight from unnecessarily reaching the onboard SD panel.

During the first year of the Aqua mission, the SD/SDSM system was operated on a weekly basis. This was followed by a bi-weekly operational frequency starting on July 7 , 2003 and then a tri-weekly operational frequency starting on January 9, 2006. If needed due to special spacecraft or sensor events, a SD/SDSM calibration can be scheduled immediately by the MCST instrument operation team (IOT) and sent to ESMO FOT for command uploads. As Aqua MODIS continues to operate beyond its design lifetime of 6 years, a minor adjustment has been made to further reduce the SD/SDSM calibration frequency. Starting from May 19, 2008, the SD/SDSM calibration without the SDS is performed on a six-week basis and the calibration with the SDS remains on a tri-weekly basis. This reduces direct solar exposure on the SD, as well as the number of SD door step motor's movements. A summary of Aqua MODIS SD/SDSM calibration events executed from instrument launch to present (June 2002 to December 2008) is provided in Table 3 .

In addition to SD/SDSM activities, lunar observations are scheduled on a near monthly basis. MODIS lunar observations are planned to track its RSB radiometric calibration stability. They are made during orbit nighttime through the instrument SV port via spacecraft roll maneuvers at nearly the same lunar phase angles: $\left[-55^{\circ}\right.$ to $\left.-56^{\circ}\right]$ for Aqua MODIS (waxing) and $\left[56^{\circ}\right.$ to $\left.55^{\circ}\right]$ for Terra MODIS (waning). The numbers of scheduled 
lunar observations made each year are also provided in Table 3. Typically, there are 8-10 lunar observations each year. In 2006, however, 2 of these lunar observations were missed due to spacecraft related events. When applying lunar observations to monitor the RSB calibration stability or changes in the long-term responses, several geometric factors, such as lunar phase angles $\left(f_{P A}\right)$, libration angles $\left(f_{L A}\right)$, and the distances between the Sun and Moon $\left(d_{S M}\right)$ and the Moon and MODIS $\left(d_{M M}\right)$, must be included. By analogy, a relative lunar calibration coefficient $\left(m_{l}^{\prime}\right)$ similar to Eqn. 1 can be expressed as

$$
m_{1}{ }^{\prime}=\frac{f_{P A} \cdot f_{L A} \cdot f_{O S}}{d n_{M o o n}^{*} \cdot d_{S M}^{2} \cdot d_{M M}^{2}}
$$

where $f_{O S}$ is an over-sample factor needed since multiple overlapping lunar scans are used. The combination of the above correction factors can be provided via a lunar model [18-19]. Together with SD observations made at an AOI of $50.2^{\circ}$ to the scan mirror, MODIS lunar observations made at an AOI of $10.2^{\circ}$ are primarily used to track and correct changes in sensor response versus scan-angle (RVS).

Since no pre-launch measurements were made to characterize the SD BRF at the SDSM's $\mathrm{SD}$ view angle and to characterize the SDS vignetting function, a series of yaw maneuvers, covering all possible viewing geometries for the SD calibration, were implemented on-orbit to mitigate this deficiency. SD and SDSM observations, made during spacecraft yaw maneuvers with and without the SDS in place, provided useful data sets to derive these key parameters [20]. As described in section 2, MODIS SWIR band observations are slightly impacted due to the out-of-band (OOB) thermal leak from MWIR and electronic crosstalk. Because of this, a correction algorithm is applied to the on-orbit calibration algorithm. The correction coefficients used to remove the thermal leak are derived and monitored on-orbit using EV observations made at nighttime. For this purpose, a special nighttime to daytime switch of the sensor operation mode has to be made, since nominal operation does not collect nighttime EV data for the RSB. This activity is performed less frequently than others (Table 3 ).

\section{ON-ORBIT PERFORMANCE}

Unlike Terra MODIS, which has experienced a number of changes in its operational configurations, Aqua MODIS has operated using the same configuration (redundant Bside) during its entire mission. The decision to use the B-side as the sensor at-launch configuration was based on the results from extensive pre-launch characterization and performance comparison of both A-side and B-side configurations. Table 4 provides an operational timeline of Aqua MODIS, from launch to present. Aqua MODIS was launched on May 04, 2002. After a series of sensor activation activities, the instrument was switched to the "science mode" on June 07,2002 . This was followed by a complete set of operational and functional tests. The instrument nadir aperture door was opened on June 24, when the first Earth view image ("First Light") was recorded. For more than 6 years, only 3 spacecraft related anomalies, which occurred shortly after launch, have 
caused MODIS to switch briefly to the safe mode (http://www.mcst.ssai.biz/mcstweb/ Formatted: Font color: Auto performace/aqua_instrument.html).

\subsection{Instrument and Focal Plane Temperatures}

MODIS on-orbit calibration is made at an end-to-end system level. The sensor response includes contributions from its optics (including scan mirror, telescope, and aft optics), detectors, and electronics. The stability of instrument and focal plane temperatures could have a direct impact on each individual detector's response and, likely, the calibration quality if no correction is applied. Figure 2 shows Aqua MODIS instrument, VIS, and NIR FPA temperatures on a scan-by-scan basis over a two- hour period (1 orbit: $98 \mathrm{~min}$ ) in 2002 (a) and 2008 (b). Excluding extreme outliers due to random telemetry noises, the temperature scan-by-scan variations, largely due to nighttime and daytime orbit differences, are typically less than $0.5 \mathrm{~K}$ (peak-to-peak) during nominal sensor operations. Using their granule (5min) averages, the orbit-to-orbit variations of Aqua MODIS VIS and NIR FPA temperatures are illustrated in Figure 3 (a) for a 3-day period (1 day: 14.4 orbits). Their long-term trends and seasonal variations, from launch to present (December, 2008), are provided in Figure 3 (b) using the daily averages. The orbit-toorbit difference (min-to-max) within a day is on the order of $1.0 \mathrm{~K}$. After more than 6 years of on-orbit operation, the instrument temperature, on average, has only increased by $2.0 \mathrm{~K}$. The seasonal variations are closely correlated to the distance between the Sun and the spacecraft and the solar beta angles of the spacecraft. The VIS and NIR FPA have no temperature control. As expected, their temperature behaviors are very similar to the instrument temperatures. The SWIR bands are co-located with the MWIR bands on the SMIR FPA, which is nominally controlled at $83 \mathrm{~K}$.

In RSB calibration algorithms (Eqns. 1 and 2), the detector responses to the SD and EV in digital numbers are corrected for the instrument temperature effect. This is necessary since the EV observations are made continuously on a scan-by-scan basis, while the SD calibration is only performed on a regular basis from weekly to tri-weekly. For VIS and NIR spectral bands, this temperature correction also includes their FPA temperature effect. For SWIR bands, there is no FPA temperature effect since the SD and EV observations are made at the same FPA temperature.

\subsection{SD On-orbit Degradation}

The MODIS SD bi-directional reflectance factor (BRF), which serves as the RSB calibration reference traceable to the NIST reflectance standard, was characterized prelaunch by the instrument vendor. Its on-orbit degradation is monitored by the SDSM at nine wavelengths between 0.412 and $0.936 \mu \mathrm{m}$, shown in Table 2, during each scheduled SD/SDSM calibration activity. To maintain RSB calibration quality, on-orbit changes in the SD BRF must be taken into account when computing calibration coefficients from sensor SD observations (Eqn. 1). Details of the methodology applied to track the SD onorbit degradation and specific implementation techniques have been reported in a number of references $[10,17]$. Fitting results of Aqua MODIS SD degradation, from launch to present, are presented in Figure 4, with normalization made relative to SDSM detector 9 (D9) at $0.936 \mu \mathrm{m}$, since the degradation at the D9 wavelength has been extremely small [21]. Fitting residuals vary from $0.2 \%$ at the long wavelength end to $0.5 \%$ at the short 
wavelength end. The normalization is applied to reduce the SDSM screen vignetting effect.

As expected, the SD on-orbit degradation shows a clear dependence on the amount of solar exposure. In addition, the degradation is strongly wavelength-dependent. There has been a relatively large one-time change in the SD degradation near day 222 (August 10, 2002). This was caused by extra solar exposure on the SD when the SD door was left open (without SD screen) for 5 days as a result of a failed command upload/execution (Table 4). On average, the Aqua MODIS SD annual degradation rate is approximately $2.6 \%$ at $0.412 \mu \mathrm{m}(\mathrm{D} 1), 1.6 \%$ at $0.466 \mu \mathrm{m}(\mathrm{D} 2), 1.0 \%$ at $0.530 \mu \mathrm{m}$ (D3), $0.8 \%$ at $0.554 \mu \mathrm{m}$ (D4), $0.4 \%$ at $0.646 \mu \mathrm{m}$ (D5), and less than $0.2 \%$ at other longer wavelengths (D6-D8). In general, the Aqua MODIS SD degradation rates are nearly identical to Terra MODIS SD degradation when both instruments are operated under the same conditions. Since its SD door was permanently set at the "open" position (on 02 July 2003), the Terra MODIS SD degradation has accelerated [10].

\subsection{Detector Noise Characterization}

Detector signal-to-noise (SNR) is a key performance parameter for earth-observing sensors, and directly impacts calibration accuracy and stability, as well as the image quality. For Aqua MODIS RSB (a total of 330 individual detectors), there are 14 inoperable detectors ( 1 in band 5 and 13 in band 6) and 3 noisy detectors (all in band 6) with most identified pre-launch and a few that occurred at launch or due to spacecraft related anomalies at mission beginning (Table 4). Since then, there have been no new noisy or inoperable detectors in RSB. Due to a large number of inoperable and noisy detectors [22], no science data products are derived from direct use of band 6. On-orbit, the RSB noise characterization is performed for each individual detector using its responses to the sunlight-illuminated SD panel during each scheduled SD calibration event. There are 50 data samples collected each scan over the SD calibration sector. Each detector's SNR is computed at different signal levels and then scaled to the typical radiance level (Ltyp) via a smooth fitting of SNRs at these levels. On a regular basis, the measured SNR values at Ltyp are compared with the specified SNR listed in Table 1, as part of the MCST tasks of monitoring sensor on-orbit performance.

Table 5 is a summary of SNR performance on an annual basis, in terms of measured SNR normalized to the specified SNR for each spectral band. Previously identified noisy and inoperable detectors are excluded from the band average. Clearly, the RSB noise characterization performance has continued to exceed the design requirements, with all the numbers in Table 5 being larger than 1 . There has been a small, but noticeable, performance degradation for bands 8 and 9 . This is primarily due to decreases of their onorbit responses over time (see section 4.5).

\subsection{Response Short-term Stability}

MODIS RSB detector short-term stability is evaluated using their response to the SD when it is fully illuminated. Normally, a total of 40 scans of fully illuminated SD observations are selected as the "sweet spot" range when computing the calibration coefficients (20 scans for each mirror side). The "sweet spot" range is the middle portion 
of the scans when the SD is fully illuminated by the Sun. In this study, the scan-by-scan calibration coefficients $\left(m_{1}\right)$ from SD observations are used to examine the detector shortterm stability. Figures 5-8 are examples for bands 10,11, 18, and 19 with results from mirror side 1 ( 3 middle detectors). As previously described, a complete RSB calibration consists of two sets of SD observations: one with and one without the SD screen (SDS) in place. Bands 10 and 11 are calibrated with the SDS and bands 18 and 19 without the SDS.

In Figure 5, band 10 detector responses are modulated by the SDS vignetting function (Eqn. 1), which introduces additional features and, therefore, impacts its short-term stability. With the SDS in place, band 10 peak-to-peak variations of its scan-by-scan calibration coefficients $\left(m_{1}\right)$ are within $\pm 0.3 \%$. It is expected that the actual detector's short-term stability, after removing the SDS effect, should be better than what has been illustrated in Figure 5. Nevertheless, the impact due to screen modulation or scan-by-scan variations on RSB calibration is still very small. This is because the calibration coefficients derived from each SD calibration event are averaged over all 20 scans within the "sweet spot" range. As expected, the short-term stability of band 11, as illustrated in Figure 6 , is very similar to band 10 . For band 18 , on the other hand, there is no SD screen effect in the detector responses. As shown in Figure 7, variations of its scan-by-scan calibration coefficients $\left(m_{l}\right)$ are typically within $\pm 0.1 \%$. Similar performance is illustrated in Figure 8 for band 19. It should be pointed out that detector short-term stability is closely related to its SNR. Detectors with smaller noise often exhibit better short-term stability.

\subsection{Response Long-term Changes}

Figure 9 (a) shows long-term changes in the responses or gains $\left(1 / m_{1}\right)$ determined from SD observations for Aqua MODIS VIS spectral bands (mirror side 1). The responses are averaged over detectors for each spectral band and normalized to their initial on-orbit values. Identified inoperable and noisy detectors are excluded from band-averaged trending. Since launch, there has been a response decrease of $23 \%$ for band 8 at $0.412 \mu \mathrm{m}$, followed by a decrease of $15 \%$ for band 9 at $0.443 \mu \mathrm{m}, 10 \%$ for band 3 at $0.469 \mu \mathrm{m}$, and $8 \%$ for band 10 at $0.488 \mu \mathrm{m}$. On average, these changes correspond to an annual decrease of approximately $3.6 \%$ for band $8,2.3 \%$ for band $9,1.6 \%$ for band 3 , and $1.2 \%$ for band 10. For the remaining VIS bands $(11,12$, and 4$)$, the response decrease has been less than $3 \%$ from launch to present, or $0.5 \%$ per year. For the VIS spectral bands, the ratios of mirror side 1 responses $\left(1 / m_{1}\right)$ to mirror side 2 responses are illustrated in Figure 9 (b). Compared to mirror side 1 , the mirror side 2 responses have experienced additional changes of $1.7 \%$ for band $8,0.8 \%$ for band $9,0.5 \%$ for band 3 , and $0.2 \%$ for band 10 over a period of more than 6 years. This wavelength dependent change (decrease) in Aqua MODIS VIS band responses is very similar to Terra MODIS, except that the mirror side differences in Aqua MODIS VIS bands have been much smaller. As a result, the Aqua MODIS VIS calibration and data product quality has been better than Terra MODIS.

The long-term changes in the NIR responses are presented in Figure 10. Bands 13 and 14 have both high (H) and low (L) gains. Trending results of $13 \mathrm{H}$ and $14 \mathrm{H}$, which are nearly 
identical to $13 \mathrm{~L}$ and $14 \mathrm{~L}$, are not included in Figure 10. It is clear that bands $15-19$ have similar behavior, with response changes less than $\pm 1.5 \%$ over the entire mission. For band $13 \mathrm{~L}$, there is a total of $3.0 \%$ increase, primarily due to changes in its electronic gain. Meanwhile, bands 1 and 2 (the $0.25 \mathrm{~km}$ resolution bands) have shown noticeably different trending behavior than other bands, with an initial decrease up to $3 \%$ and then an increase of approximately $5 \%$ in their responses (relative to their initial values). Response trending results from lunar observations also show similar behavior for bands 1 and 2 . The system level detector response, derived from SD and lunar observations, is a measure of changes in optics, detector, and electronic gains. Small increases in the responses of some NIR bands are likely due to a combined effect of detector and electronic gain changes. For all the NIR bands, the mirror side differences are less than $0.1 \%$, a factor of 3 smaller than Terra MODIS NIR bands over the same time period.

Bands 5-7 and 26 are the SWIR bands. They are co-located with the mid-wave infrared (MWIR) bands on the SMIR FPA, which is normally operated at 83K. As shown in Figure 11, the changes in the responses of bands 5, 7, and 26 have been very small, less than $1.5 \%$ over the entire mission, with practically no mirror side difference. Band 6 has similar behavior except for the changes at the mission beginning due to spacecraft related events (Table 4). These events sent MODIS to its safe mode and required a restart for the sensor normal operation, which led to different numbers of noisy and inoperable detectors in band 6. Clearly, the constantly controlled SMIR FPA temperature has contributed to the excellent long-term stability for the SWIR bands.

\subsection{Lunar Calibration}

In addition to SD calibration, MODIS views the Moon through its space view (SV) port. As listed in Table 3, there are 8-10 regularly scheduled lunar observations each year. Presented in Figure 12 (a) is the long-term response trending, $1 / m_{l}{ }^{\prime}$ (Eqn. 3), for Aqua MODIS VIS spectral bands derived from their lunar observations with mirror side 1 . Similar to SD response trending in Figure 9 (a), the responses in Figure 12 (a) are also normalized to their initial on-orbit values. In general, the lunar response trending matches well with the SD response, except the degradation rate from lunar observations (at lunar view $\mathrm{AOI}$ ) is slightly higher than that from SD observations for several spectral bands. By comparison, the responses at lunar AOI have decreased $34 \%$ for band 8 at $0.412 \mu \mathrm{m}$, $20 \%$ for band 9 at $0.443 \mu \mathrm{m}, 12 \%$ for band 3 at $0.469 \mu \mathrm{m}$, and $8 \%$ for band 10 at $0.488 \mu \mathrm{m}$. For bands 11,12 , and 4 , the changes since launch have been less than $2 \%$.

As illustrated in Figure 12 (b), mirror side 2 responses have additional changes of approximately $2.3 \%$ for band $8,1.5 \%$ for band $9,1.0 \%$ for band 3 , and $0.5 \%$ for band 10 , compared to mirror side 1 responses. The variations of mirror side differences derived from lunar observations are noticeably larger compared to SD observations. This is primarily due to the lunar surface's non-uniformity. For most RSB bands, the differences between SD and lunar observations and mirror side differences are small. Since the RSB calibration (Eqn. 1) is mirror side dependent, the mirror side difference is captured by the SD calibration. For each mirror side, the difference between the degradation rates derived from SD and from lunar observations is used to track the changes in sensor response versus scan angle (RVS). 


\section{LESSONS AND CHALLENGES}

Many lessons from Terra MODIS RSB calibration, pre-launch to post-launch, have greatly benefitted Aqua MODIS:

- Lessons learned from Terra MODIS SWIR bands thermal leak and electronic crosstalk characterization have led to more effective pre-launch mitigation efforts in dealing with the same problem in Aqua MODIS. Largely reduced thermal leak and electronic crosstalk effects in Aqua MODIS SWIR bands has resulted in noticeably improved performance for the on-orbit correction algorithm, and, therefore, the data product quality [15].

- The Terra MODIS on-orbit experience in characterizing the SD BRF, including the SDSM viewing angle, and SDS vignetting function (not characterized pre-launch) have led to improvements for similar work in Aqua MODIS, including planning and implementation of yaw maneuvers and special data analysis [20]

- MODIS SDSM was built with a misalignment (mechanically fixed) in its Sun view path, which caused undesirable ripples in the responses. These ripples significantly limited the effectiveness of the SDSM's capability to track SD on-orbit degradation. Shortly after Terra launch, many efforts were made to better characterize the effect and associated impact on SD degradation quality. As a result, an alternative approach of normalizing other SDSM detectors to detector 9 was developed and applied to Terra MODIS. This has been proven to be very effective, since the SD has little degradation at the D9 wavelength and all SDSM detectors have similar ripples [16]. Because of this, no additional effort is required to make the Aqua MODIS SDSM work effectively in tracking its SD on-orbit degradation.

- Similar efforts and tools developed for Terra MODIS lunar observations have been directly applied to Aqua MODIS, including the lunar observation planning tool, required spacecraft roll maneuvers to make the lunar observations at the same phase angles, and determination of lunar viewing geometric factors [19].

- Experience of on-orbit changes in Terra RSB, especially the VIS spectral bands, response versus scan angle (RVS), and the challenging effort needed to track and update the changes for the L1B calibration algorithm have better prepared similar work in Aqua MODIS.

On the other hand, there have been some lessons learned from Aqua MODIS RSB calibration and characterization that in turn have helped Terra MODIS. For MODIS RSB, the calibration accuracy requirement is $\pm 2 \%$ in reflectance. Uncertainty analysis indicates that a major contributor or a limiting factor to the RSB calibration is the SD BRF [23]. It, by itself, has three components: pre-launch characterization uncertainty, potential change from pre-launch characterization to first time orbit use of the SDSM, and on-orbit degradation monitoring uncertainty. As some uncertainty components were fixed based on pre-launch characterization, accurately tracking sensor on-orbit responses (optics, electronics, and detectors), including SD degradation, and promptly updating changes needed to maintain the L1B calibration and data product quality are constant and challenging tasks undertaken by the MODIS Characterization Support Team (MCST). 
Additional effort is needed for MODIS to track changes in sensor response as a function of scan angle. Some science data products, such as ocean color, need sensor polarization parameters for atmospheric correction. These parameters are typically derived from prelaunch measurements and it is extremely hard to track their on-orbit changes [24].

As expected, lessons from both Terra and Aqua MODIS have and will continue to provide valuable information for the design and development of future earth observing sensors, such as the NPP and NPOESS VIIRS, the GOES-R ABI, and the LDCM OLI. VIIRS, essentially a MODIS follow-on sensor, will use a nearly identical SD/SDSM calibration system for the VIS/NIR/SWIR spectral bands with improvements made to correct the SDSM deficiency and to make pre-launch measurements of the SD screen and SDSM Sun view screen vignetting function. Adding an on-board SD for ABI is a major step towards improving on-orbit calibration quality for the operational geostationary sensors. MODIS on-orbit SD degradation tracking capability has been added to OLI, the solar reflective sensor of LDCM, developed to extend the existing Landsat missions. In addition to sensor design and selection of its on-board calibration capability, MODIS RSB calibration activities, methodologies, and results have been extensively used and referenced to address the need for future sensors with significant improvements in the solar reflective spectral regions. The Climate Absolute Radiance and Refractivity Observatory (CLARREO) mission is such an example. It is recommended in the NRC Decadal Survey as a key component for the future climate observing system, with shared responsibility by both NASA and NOAA. It is currently in the early plan and design phase, aimed at producing highly accurate and spectrally resolved on-orbit SI traceable benchmark measurements for climate studies.

\section{CONCLUSIONS}

Excluding a few small data gaps due to spacecraft and sensor operational anomalies, Aqua MODIS has been making continuous global observations for more than six years since its nadir aperture door opened on June 24, 2002 (sensor "first light"). Dedicated effort by the MODIS Science Team and Characterization Support Team (MST and MCST) has greatly contributed to the mission's success. Excluding noisy or inoperable detectors (nearly all in band 6) identified pre-launch and shortly after launch, all RSB detectors have excellent short-term stability, meeting noise characterization and design requirements. The changes in sensor responses, typically larger in the VIS spectral region, are constantly monitored and corrected in the calibration algorithm. In general, the changes in Aqua MODIS RSB, as well as the SD degradation, are relatively small compared to its predecessor, Terra MODIS. In addition, Aqua MODIS has a much smaller electronic crosstalk in its SWIR bands and shows a much smaller mirror side difference in sensor responses for all spectral bands. Consequently, Aqua MODIS has demonstrated an overall better performance than Terra MODIS. To this date, Aqua MODIS continues to operate normally and produce high quality measurements of radiances and reflectance factors needed as input for many science data products. Lessons learned from MODIS operation, calibration, algorithm design, and performance have provided useful references for future earth observing sensors. 


\section{ACKNOWLEDGMENTS}

The authors would like to thank members of the MODIS Characterization Support Team and the Raytheon SBRS MODIS instrument team for their technical assistance and contributions made during Aqua pre-launch calibration and characterization and through its on-orbit operation. We also appreciate special assistance provided by A. Angal and J. Dodd in preparing this manuscript.

\section{REFERENCES}

1. C.L. Parkinson, "Aqua: An Earth-Observing Satellite Mission to Examine Water and Other Climate Variables," IEEE Trans. Geosci. Remote Sensing, 41, 173-183, 2003.

2. W.L. Barnes and V.V. Salomonson, "MODIS: A global image spectroradiometer for the Earth Observing System," Critical Reviews of Optical Science and Technology, CR47, 285-307, 1993.

3. W.L. Barnes, V.V. Salomonson, B. Guenther, and X. Xiong, "Development, Characterization, and Performance of the EOS MODIS Sensors," Proceedings of SPIE - Earth Observing Systems VIII, 5151, 337-345, 2003.

4. V.V. Salomonson, W.L. Barnes, X. Xiong, S. Kempler, and E. Masuoka, "An Overview of the Earth Observing System MODIS Instrument and Associated Data Systems Performance," Proceedings of IGARSS, 2002.

5. C.O. Justice, E. Vermote, J.R.G. Townshend, R. Defries, D.P. Roy, D.K. Hall, V.V. Salomonson, J.L. Privette, G. Riggs, A. Strahler, W. Lucht, R.B. Myneni, P. Lewis, and M.J. Barnsley, "The Moderate Resolution Imaging Spectroradiometer (MODIS): Land Remote Sensing for Global Change Research," IEEE Trans. Geosci. Remote Sensing, 36, 1228-1249, 1998.

6. W.E. Esaias, M.R. Abbott, I. Barton, O.W. Brown, J.W. Campbell, K.L. Carder, D.K. Clark, R.L. Evans, F.E. Hoge, H.R. Gordon, W.P. Balch, R. Letelier, and P.J. Minnett, "An Overview of MODIS Capabilities for Ocean Science Observations," IEEE Trans. Geosci. Remote Sensing, 36, 1250-1265, 1998.

7. M.D. King, W.P. Menzel, Y.J. Kaufman, D. Tanre, B.C. Gao, S. Platnick, S.A. Ackerman, L.A. Remer, R. Pincus, and P.A. Hubanks, "Cloud and Aerosol Properties, Precipitable Water, and Profiles of Temperature and Water Vapor from MODIS," IEEE Trans. Geosci. Remote Sensing, 41, 442-458, 2003.

8. X. Xiong, K. Chiang, J. Esposito, B. Guenther, and W. Barnes, "MODIS On-orbit Calibration and Characterization," Motrologia, 40, 89-92, 2003.

9. W.L. Barnes, T.S. Pagano, and V.V. Salomonson, "Prelaunch characteristics of the Moderate Resolution Imaging Sectroradiometer (MODIS) on EOS-AM1," IEEE Trans. Geosci. Remote Sensing, 36, 1088-1100, 1998.

10. X. Xiong, J. Sun, W. Barnes, V. Salomonson, J. Esposito, H. Erives, and B. Guether, "Multiyear on-orbit calibration and performance of Terra MODIS reflective solar bands," IEEE Trans. Geosci. Remote Sensing, vol. 45, no. 4, 879-889, 2007. 
11. X. Xiong, K. Chiang, A. Wu, W. Barnes, B. Guenther, and V. Salomonson, "Multiyear on-orbit calibration and performance of Terra MODIS thermal emissive bands," IEEE Trans. Geosci. Remote Sensing, vol. 46, no. 6, 1790-1803, 2008.

12. X. Xiong, N. Che, and W. Barnes, "Terra MODIS On-orbit Spatial Characterization and Performance", IEEE Trans. Geosci. Remote Sensing 43, 355-365, 2005.

13. X. Xiong, N. Che, and W.L. Barnes, "Terra MODIS On-orbit Spectral Characterization and Performance", IEEE Trans. on Geosci. Remote Sensing, vol. 44, no. 8, 2198-2206, 2006.

14. X. Xiong, B. Wenny, A. Wu, W.L. Barnes, and V. Salomonson, “Aqua MODIS Thermal Emissive Bands On-orbit Calibration, Characterization, and Performance," IEEE Trans. Geosci. Remote Sensing, 47(3), 803-814, 2009.

15. X. Xiong, K. Chiang, F. Adimi, W. Li, H. Yatagai, and W.L. Barnes, "MODIS Correction Algorithm for Out-of-band Response in the Short-wave IR Bands", Proceedings of SPIE - Sensors, Systems, and Next Generation of Satellites VII, 5234, 605-613, 2004.

16. X. Xiong, J. Sun, J. Esposito, X. Liu, W.L. Barnes, and B. Guenther, “On-orbit Characterization of a Solar Diffuser's Bi-directional Reflectance Factor Using Spacecraft Maneuvers," Proceedings of SPIE - Earth Observing Systems VIII, 5151, 375-383, 2003.

17. X. Xiong, H. Erives, S. Xiong, X. Xie, J. Esposite, J. Sun, and W.L. Barnes, "Performance of Terra MODIS Solar Diffuser and Solar Diffuser Stability Monitor," Proceedings of SPIE - Earth Observing Systems X, Vol. 5882, 58820S, doi:10.1117/12.615334, 2005.

18. H. Kieffer and T. Stone, "The Spectral Irradiance of the Moon," The Astronomical Journal, 129, 2887-2901, 2005.

19. J. Sun, X. Xiong, W. Barnes, and B. Guether, "MODIS reflective solar bands on-orbit lunar calibration," to IEEE Trans. Geosci. Remote Sensing, vol. 45, no. 7, 2383-2393, 2007.

20. X. Xie, X. Xiong, D. Moyer, J. Sun, X. Liu, and W.L. Barnes, "Analysis of MODIS Solar Diffuser Screen Vignetting Function," Proceedings of SPIE - Earth Observing Systems $X$, Vol. 5882, 58820T, doi:10.1117/12.615394, 2005.

21. X. Xiong, W.L. Barnes, X. Xie, and V.V. Salomonson, "On-orbit Performance of the Aqua MODIS Onboard Calibrators," Proceedings of SPIE - Sensors, Systems, and Next Generation of Satellites IX, 5978, 59780U, doi:10.1117/12.627619, 2005.

22. X. Xiong, W.L. Barnes, B. Guenther, and R.E. Murphy, "Lessons Learned From MODIS," Adv. Space Res., 32(11), 2107-2112, 2003.

23. J. Esposito, X. Xiong, A. Wu, J. Sun, and W.L. Barnes, "MODIS Reflective Solar Bands Uncertainty Analysis," Proceedings of SPIE - Earth Observing Systems IX, 5542, 448-458, 2004.

24. J. Sun and X. Xiong, "MODIS Polarization Sensitivity Analysis," IEEE Transactions on Geoscience and Remote Sensing, Vol. 45, No. 9, 2875-2885, 2007. 
TABLE 1 MODIS REFlective SOLAR BAND SPECIFICATIONS

\begin{tabular}{cccccccc}
\hline Band & CW & BW & IFOV & FPA & Ltyp & Lmax & SNR \\
\hline 1 & 0.645 & 50 & 0.25 & NIR & 21.8 & 685 & 128 \\
2 & 0.858 & 35 & 0.25 & NIR & 24.7 & 285 & 201 \\
\hline 3 & 0.469 & 20 & 0.5 & VIS & 35.3 & 593 & 243 \\
4 & 0.555 & 20 & 0.5 & VIS & 29.0 & 518 & 228 \\
5 & 1.240 & 20 & 0.5 & SMIR & 5.4 & 110 & 74 \\
6 & 1.640 & 24 & 0.5 & SMIR & 7.3 & 70 & 275 \\
7 & 2.130 & 50 & 0.5 & SMIR & 1.0 & 22 & 110 \\
\hline 8 & 0.412 & 15 & 1.0 & VIS & 44.9 & 175 & 880 \\
9 & 0.443 & 10 & 1.0 & VIS & 41.9 & 133 & 838 \\
10 & 0.488 & 10 & 1.0 & VIS & 32.1 & 101 & 802 \\
11 & 0.531 & 10 & 1.0 & VIS & 27.9 & 82 & 754 \\
12 & 0.551 & 10 & 1.0 & VIS & 21.0 & 64 & 750 \\
13 & 0.667 & 10 & 1.0 & NIR & 9.5 & 32 & 910 \\
14 & 0.678 & 10 & 1.0 & NIR & 8.7 & 31 & 1087 \\
15 & 0.748 & 10 & 1.0 & NIR & 10.2 & 26 & 586 \\
16 & 0.869 & 15 & 1.0 & NIR & 6.2 & 16 & 516 \\
17 & 0.905 & 30 & 1.0 & NIR & 10.0 & 185 & 167 \\
18 & 0.936 & 10 & 1.0 & NIR & 3.6 & 256 & 57 \\
19 & 0.940 & 50 & 1.0 & NIR & 15.0 & 189 & 250 \\
26 & 1.375 & 30 & 1.0 & SMIR & 6.0 & 90 & 150 \\
\hline
\end{tabular}

$\mathrm{CW}$ : Center Wavelengths in $\mu \mathrm{m}$;

BW: Bandwidths in nm;

IFOV: Instantaneous Field of View (nadir) in km;

FPA: Focal Plane Assembly;

Ltyp/Lmax: Typical/Maximum Radiance in W/m²/ $/ \mu \mathrm{m} / \mathrm{sr} ; \quad$ SNR: Signal to Noise Ratio.

TABle 2 Center Wavelengths of SDSM Detectors AND Matching MODIS SPECTRAL BANDS

\begin{tabular}{cc|cc}
\hline SDSM Detectors & CW & MODIS Bands & CW \\
\hline D1 & 0.412 & 8 & 0.412 \\
D2 & 0.466 & 3 & 0.469 \\
D3 & 0.530 & 11 & 0.531 \\
D4 & 0.554 & 4 & 0.555 \\
D5 & 0.646 & 1 & 0.645 \\
D6 & 0.747 & 15 & 0.748 \\
D7 & 0.857 & 2 & 0.858 \\
D8 & 0.904 & 17 & 0.905 \\
D9 & 0.936 & 18 & 0.936 \\
\hline
\end{tabular}

CW: Center Wavelengths in $\mu \mathrm{m}$ 
TABlE 3 Number of MODIS RSB CALIBRATION ACTIVITIES

\begin{tabular}{c|ccccccc}
\hline Calibration Activity & $\mathbf{2 0 0 2}$ & $\mathbf{2 0 0 3}$ & $\mathbf{2 0 0 4}$ & $\mathbf{2 0 0 5}$ & $\mathbf{2 0 0 6}$ & $\mathbf{2 0 0 7}$ & $\mathbf{2 0 0 8}$ \\
\hline SD/SDSM & 27 & 39 & 27 & 26 & 17 & 18 & 16 \\
Lunar Observations & 5 & 9 & 10 & 9 & 9 & 7 & 9 \\
NTDM collects & 3 & 1 & 2 & 2 & 2 & 2 & 2 \\
\hline
\end{tabular}

TABle 4 Aqua MODIS Key Operational Configurations and Events

\begin{tabular}{lll}
\hline \multicolumn{2}{c}{ Date } & \multicolumn{1}{c}{ Description } \\
\hline June 07, 2002 & $(2002158)$ & Sensor switched to science mode using B-side electronics \\
June 24, 2002 & $(2002175)$ & Opening of nadir aperture door ("First light") \\
June 27, 2002 & $(2002178)$ & Spacecraft CTC swap (sensor returned to safe mode) \\
July 01, 2002 & $(2002182)$ & Opening of nadir aperture door \\
July 02, 2002 & $(2002183)$ & Sensor switched back to science mode \\
July 29, 2002 & $(2002210)$ & Spacecraft entered safe mode (sensor returned to safe mode) \\
Aug. 05, 2002 & $(2002217)$ & Opening of nadir aperture door \\
Aug. 06, 2002 & $(2002218)$ & Sensor switched back to science mode \\
Aug. 09, 2002 & $(2002221)$ & A command drop (SD door left open for 5 days) \\
Sept. 12, 2002 & $(2002255)$ & Spacecraft entered safe mode (sensor returned to safe mode) \\
Sept. 12, 2002 & $(2002255)$ & Sensor switched back to science mode \\
Dec. 02, 2007 & $(2007336)$ & Spacecraft solid state recorder anomaly, SW fix implemented \\
\hline
\end{tabular}

Table 5 Reflective Solar Bands Signal-to-Noise Ratio (NORMALIZED TO SPECIFICATIONS LIST IN TABLE 1)

\begin{tabular}{ccccccc}
\hline Band & 2002 & 2003 & 2004 & 2005 & 2007 & 2008 \\
\hline 1 & 1.55 & 1.52 & 1.54 & 1.55 & 1.56 & 1.57 \\
2 & 2.54 & 2.51 & 2.54 & 2.56 & 2.58 & 2.64 \\
3 & 1.32 & 1.30 & 1.29 & 1.28 & 1.27 & 1.24 \\
4 & 1.42 & 1.40 & 1.41 & 1.41 & 1.41 & 1.41 \\
5 & 2.05 & 2.04 & 2.04 & 2.02 & 2.00 & 2.00 \\
6 & 1.66 & 1.67 & 1.61 & 1.66 & 1.75 & 1.73 \\
7 & 1.40 & 1.40 & 1.40 & 1.41 & 1.41 & 1.41 \\
8 & 1.27 & 1.25 & 1.23 & 1.19 & 1.14 & 1.06 \\
9 & 1.84 & 1.81 & 1.79 & 1.76 & 1.74 & 1.68 \\
10 & 1.95 & 1.92 & 1.90 & 1.89 & 1.88 & 1.86 \\
11 & 2.29 & 2.26 & 2.27 & 2.26 & 2.27 & 2.27 \\
12 & 2.03 & 2.03 & 2.02 & 2.01 & 2.02 & 2.02 \\
13 & 1.58 & 1.58 & 1.61 & 1.62 & 1.63 & 1.62 \\
14 & 1.45 & 1.43 & 1.44 & 1.44 & 1.44 & 1.44 \\
15 & 2.68 & 2.63 & 2.67 & 2.66 & 2.75 & 2.76 \\
16 & 2.79 & 2.80 & 2.81 & 2.81 & 2.84 & 2.85 \\
17 & 2.20 & 2.21 & 2.21 & 2.19 & 2.21 & 2.21 \\
18 & 1.60 & 1.59 & 1.60 & 1.59 & 1.60 & 1.61 \\
19 & 2.03 & 2.04 & 2.04 & 2.04 & 2.04 & 2.04 \\
26 & 1.85 & 1.85 & 1.87 & 1.86 & 1.89 & 1.89 \\
\hline
\end{tabular}




\section{Figure Captions}

Figure 1. MODIS scan cavity and on-board calibrators

Figure 2. Aqua MODIS instrument, VIS, and NIR FPA scan-by-scan temperatures over a 2-hour period: (a) from 2002271.0000-20028271.0155; (b) from 2008271.0000- 2008271.0155

Figure 3. Aqua MODIS instrument, VIS, and NIR FPA temperatures: (a) granule averaged over a 3-day period from 2008016.0000-2008019.2355; (b) daily averaged long-term trend from launch to December 2008

Figure 4. Solar diffuser on-orbit degradation: (a) degradation as a function of time and (b) yearly accumulated degradation as a function of wavelengths

Figure 5. Band 10 calibration coefficients $\left(m_{1}\right)$ on a scan-by-scan basis (detector 4-6, mirror side 1) with data from SD calibration event on 2003104 (SDS in place)

Figure 6. Band 11 calibration coefficients $\left(m_{1}\right)$ on a scan-by-scan basis (detector 4-6, mirror side 1) with data from SD calibration event on 2003104 (SDS in place)

Figure 7. Band 18 calibration coefficients $\left(m_{1}\right)$ on a scan-by-scan basis (detector 4-6, mirror side 1) with data from SD calibration event on 2003104 (no SDS)

Figure 8. Band 19 calibration coefficients $\left(m_{1}\right)$ on a scan-by-scan basis (detector 4-6, mirror side 1) with data from SD calibration event on 2003104 (no SDS)

Figure 9. Long-term band averaged response $\left(1 / m_{1}\right)$ trending of Aqua MODIS VIS spectral bands from SD observations: (a) response from mirror side 1; (b) ratio of mirror side 1 to mirror side 2 response

Figure 10. Long-term band averaged response (1/ $\left.m_{1}\right)$ trending of Aqua MODIS NIR spectral bands from SD observations: (a) response from mirror side 1; (b) ratio of mirror side 2 to mirror side 1 response

Figure 11. Long-term band averaged response $\left(1 / m_{1}\right)$ trending of Aqua MODIS SWIR spectral bands from SD observations: (a) response from mirror side 1; (b) ratio of mirror side 2 to mirror side 1 response

Figure 12. Long-term band averaged response ( $\left.1 / m_{1}\right)$ trending of Aqua MODIS VIS spectral bands from lunar observations: (a) response from mirror side 1; (b) ratio of mirror side 2 to mirror side 1 response 
Figure 1

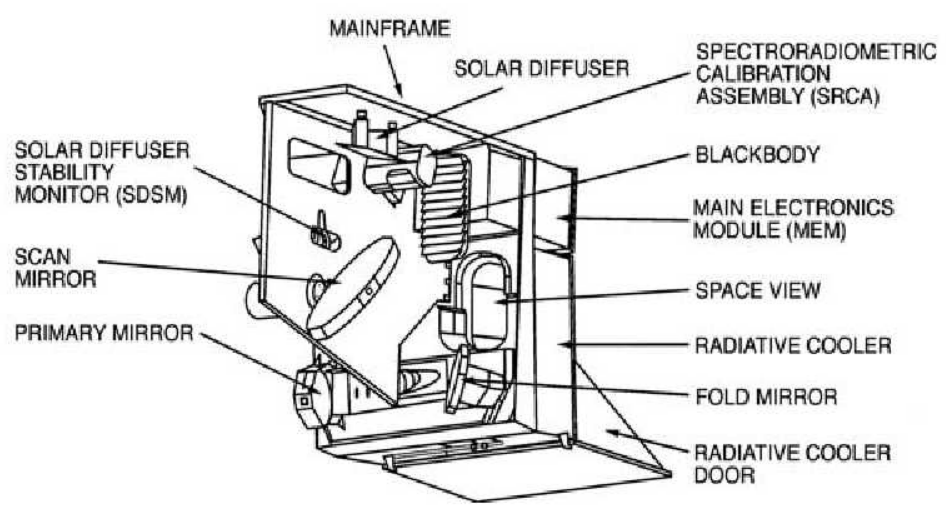


Figure 2

(a)

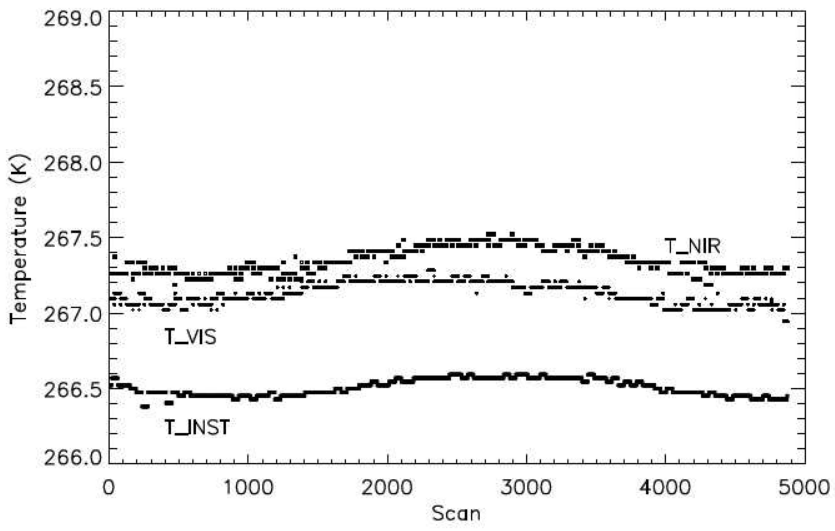

(b)

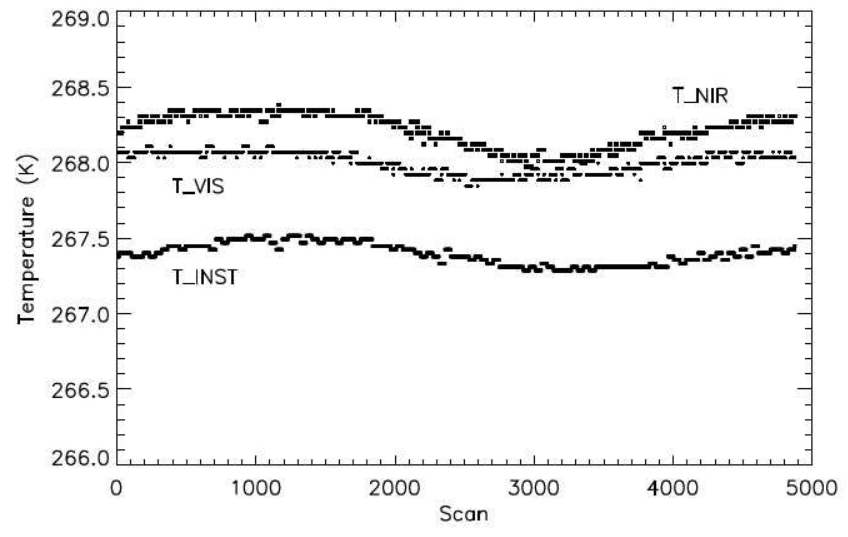


Figure 3

(a)

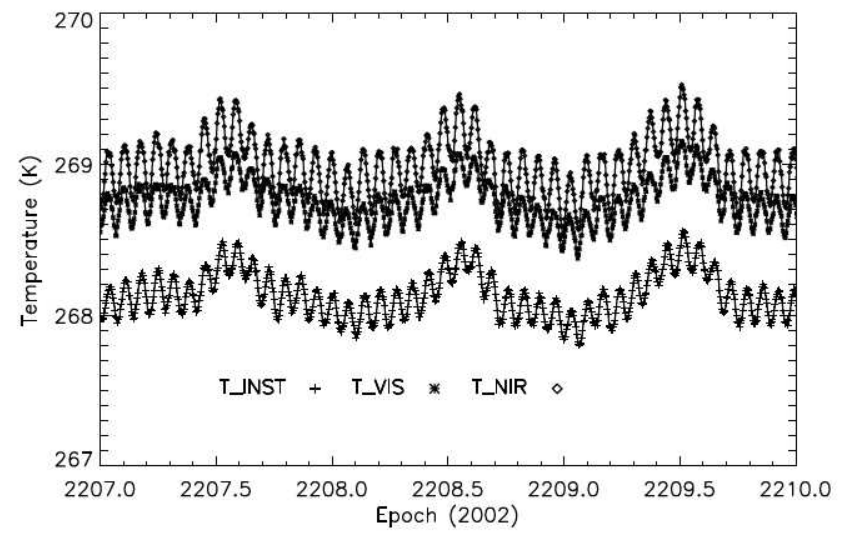

(b)

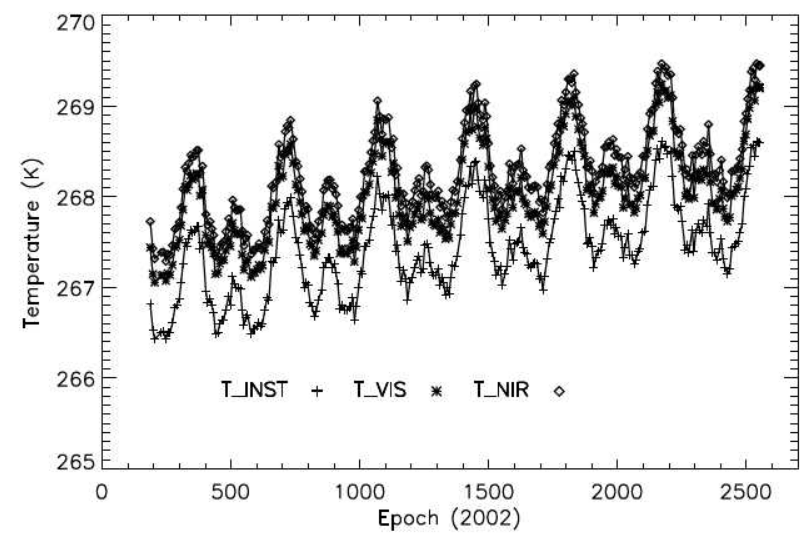


Figure 4

(a)

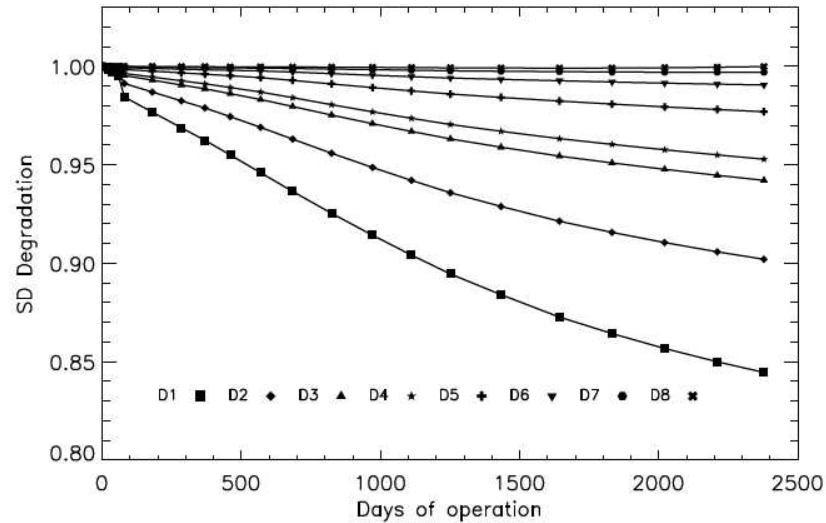

(b)

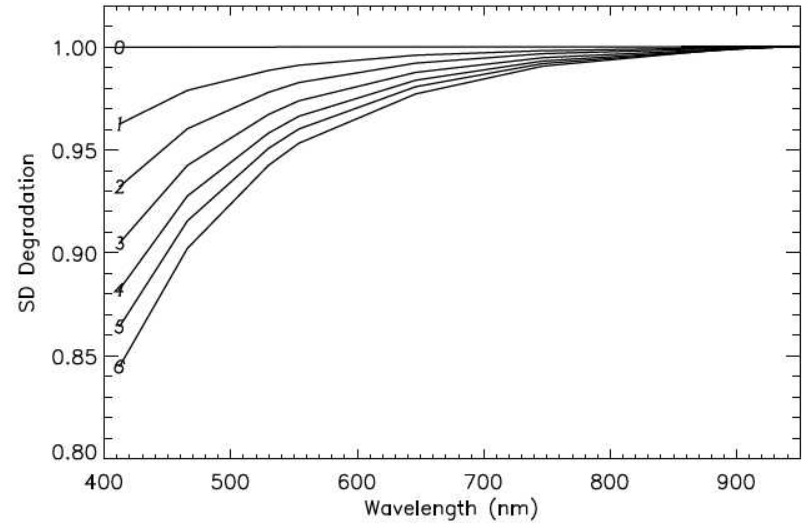


Figure 5

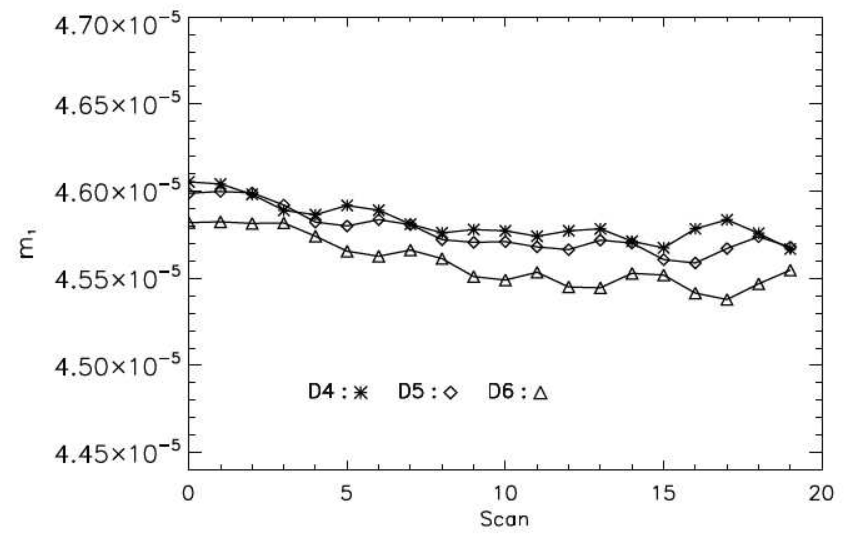

Figure 6

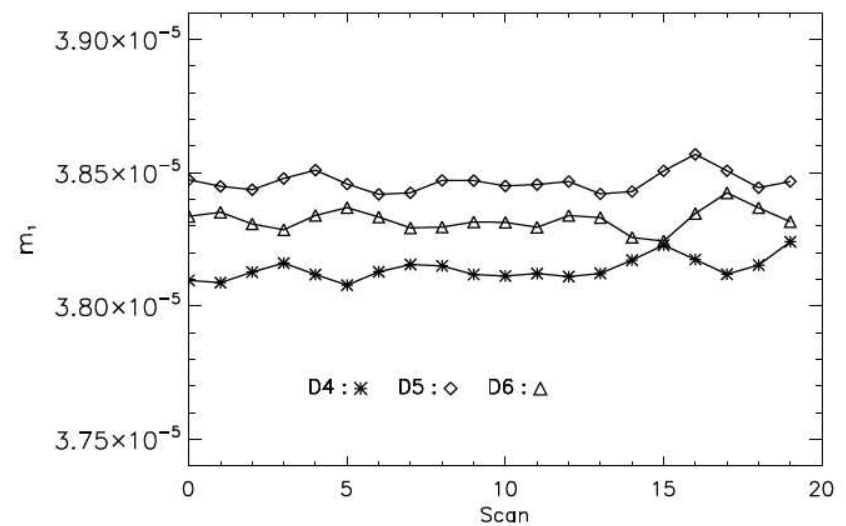


Figure 7

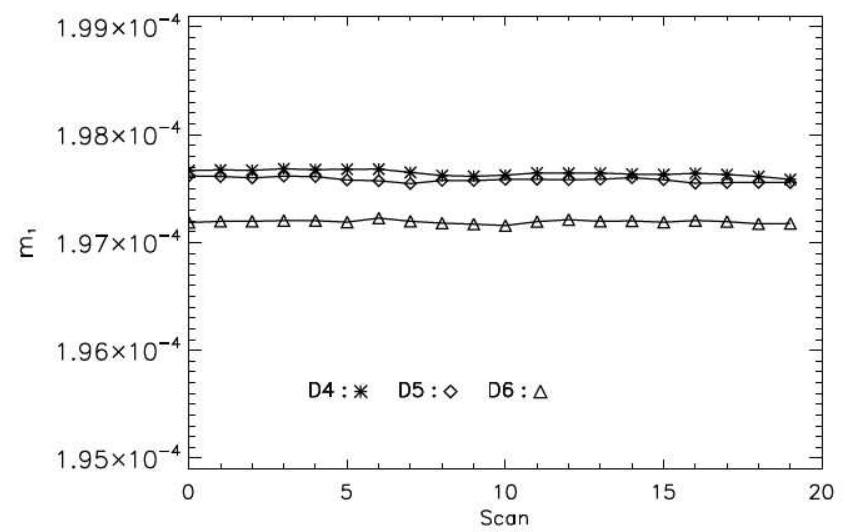

Figure 8

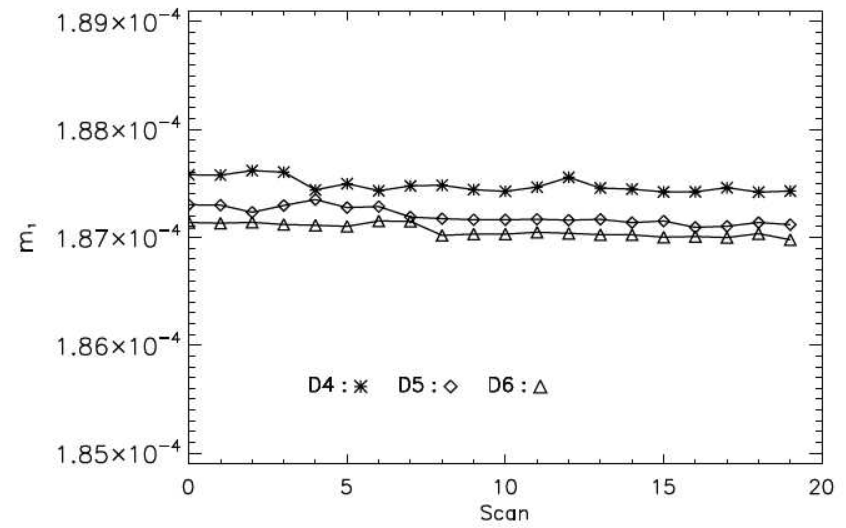


Figure 9

(a)

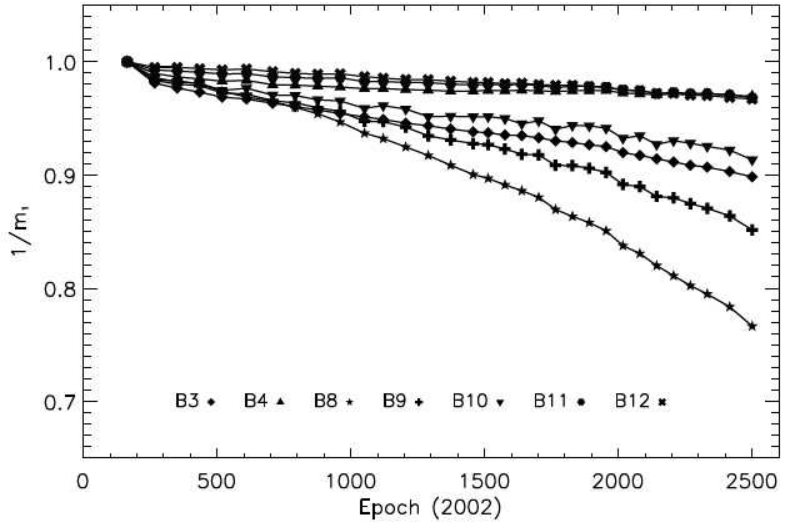

(b)

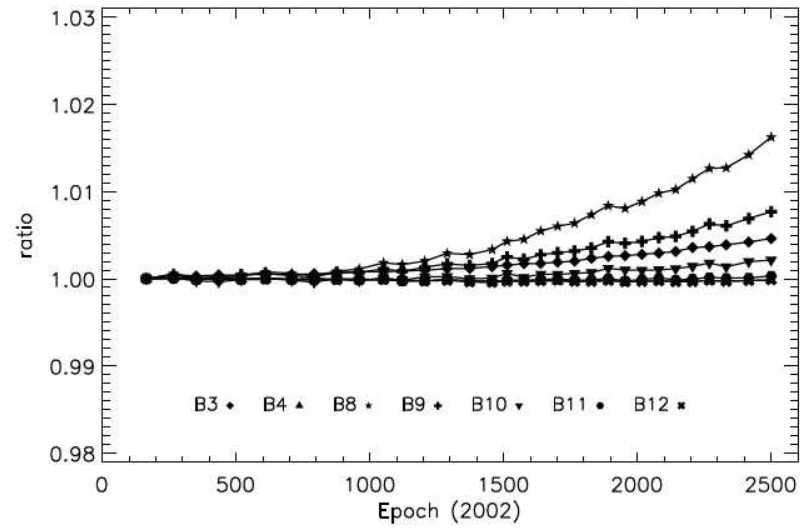


Figure 10

(a)

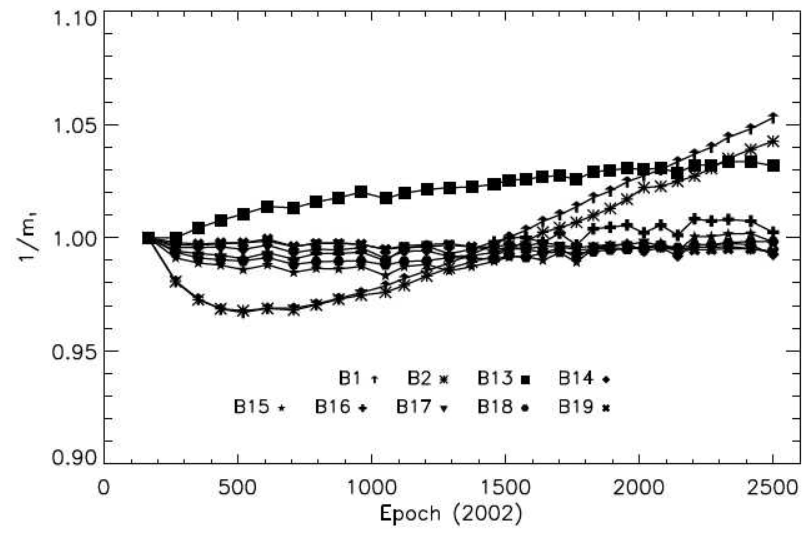

(b)

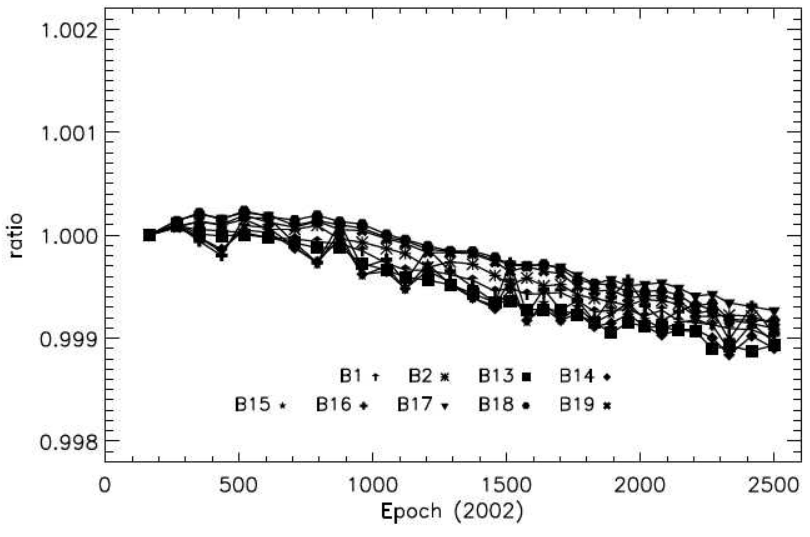


Figure 11

(a)

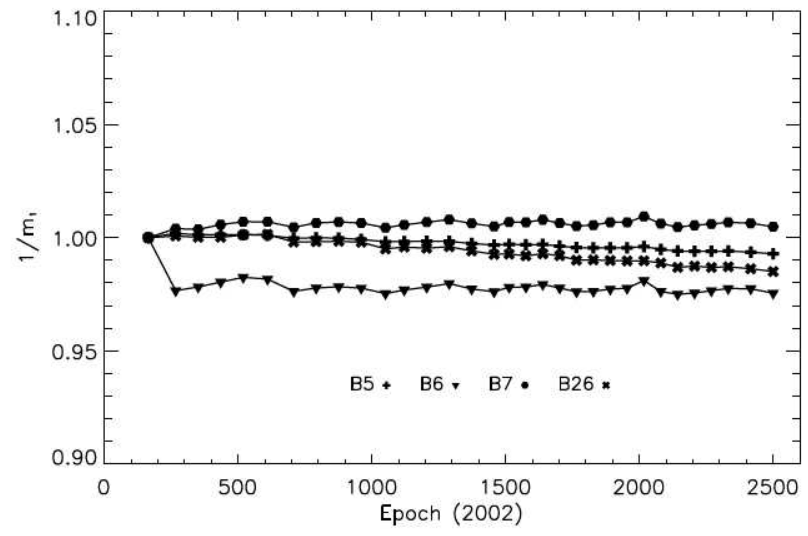

(b)

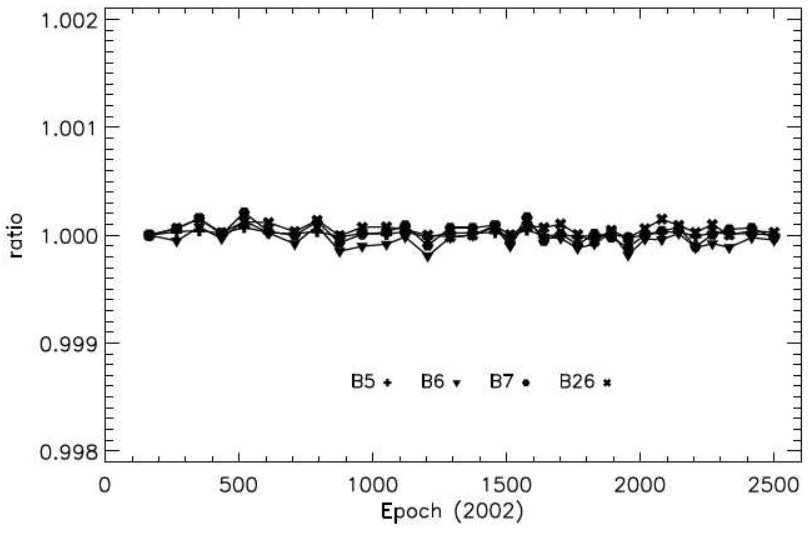


Figure 12

(a)

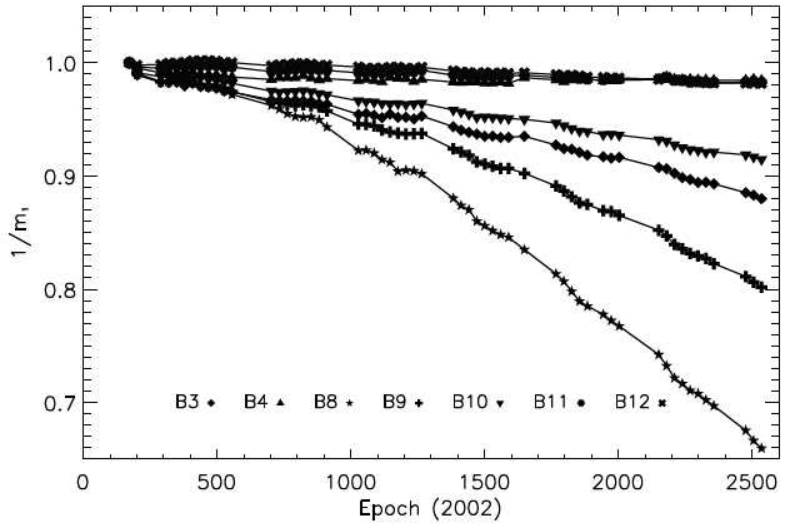

(b)

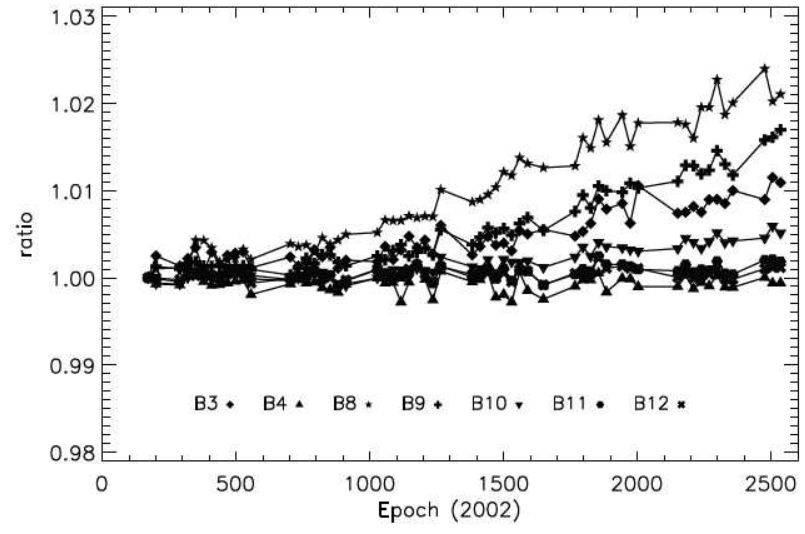

